\title{
Conceptual Design of the Mu2e Production Solenoid
}

\author{
V. V. Kashikhin ${ }^{1}$, G. Ambrosio, N. Andreev, M. Lamm, N. V. Mokhov, T. H. Nicol, T. M. Page, V. Pronskikh
}

\begin{abstract}
The Muon-to-Electron conversion experiment (Mu2e), under development at Fermilab, seeks to detect direct muon to electron conversion to provide evidence for a process violating muon and electron lepton number conservation that cannot be explained by the Standard Model of Particle Physics. The required magnetic field is produced by a series of superconducting solenoids. This paper describes the conceptual design of the $5 \mathrm{~T}, 4 \mathrm{~m}$ long solenoid with $1.7 \mathrm{~m}$ bore with the emphasis on the electromagnetic and structural analyses.
\end{abstract}

Index Terms - Electromagnetic analysis, cryogenics, solenoid, superconducting magnets.

\section{INTRODUCTION}

$\mathrm{T}^{\mathrm{s}}$ The Mu2e magnet system consists of three large 1 superconducting solenoids [1]. The first in the chain of magnets is the Production Solenoid (PS) whose role is to collect and focus pions and muons generated in interactions of an $8-\mathrm{GeV}$ proton beam with a tilted high- $\mathrm{Z}$ target, by supplying a peak axial field between $4.6 \mathrm{~T}$ and $5.0 \mathrm{~T}$ and an axial field gradient of about $1 \mathrm{~T} / \mathrm{m}$ within a $1.5 \mathrm{~m}$ warm bore.

The PS is a challenging magnet because of the relatively high magnetic field and a harsh radiation environment that requires the state-of-the-art conductor both in terms of the current-carrying capacity and structural strength. The PS coil is protected by a massive Heat and Radiation Shield (HRS). An extensive simulation effort has been carried out to optimize the shield parameters and get the radiation load below the tolerable levels with a sufficient safety margin [2].

The heat extraction scheme based on the heat conduction through a system of thermal bridges and plates connected to the thermal siphon tubes has been proposed and numerically evaluated [3] for the actual distribution of the heat depositions inside the cold mass. It was found that the required thermal margin is preserved at all conditions with an ample allowance for the heat deposition uncertainties.

The next design iteration is focused on accounting of the actual electromagnetic properties of the HRS material that has an impact on the forces acting on the cold mass during different operating scenarios, and the design of the cryostat suspension system.

The detailed description of the PS conceptual design is reported elsewhere [1], [4]. This paper focuses on the selected parameters of the cable and insulation and recent updates to the electromagnetic, structural and cryostat support designs.

Manuscript received October 9, 2012. (This work was supported in part by Fermi Research Alliance under the U.S. Department of Energy Contract DEAC02-07CH11359).

Authors are with Fermi National Accelerator Laboratory, Batavia, IL 60510 USA (vadim@fnal.gov).

\section{CABLE AND INSULATION}

\section{A. Cable}

In order to reduce the dynamic heat load on the cryogenic system due to the radiation and increase the coil thermal margin, the conductor employs aluminum stabilizer that is placed around the NbTi Rutherford type cable by either conforming or hot extrusion. Such cable technology was used in nearly every large detector solenoid built in the past decades with the most recent examples being ATLAS Central Solenoid, ATLAS Toroids and CMS [5]-[7].

The PS cable cross-section is shown in Fig. 1 and the cable parameters are summarized in Table I. A high-strength $\mathrm{Al}$ alloy with a sufficiently large RRR is necessary for the cable stabilizer. An extensive study of various precipitation hardening $\mathrm{Al}$ alloys was performed during the Atlas Central Solenoid R\&D [8]-[9]. The material selected for the PS cable stabilizer is the $5 \mathrm{~N} \mathrm{Al}$ doped with $0.1 \mathrm{wt} \% \mathrm{Ni}$ in combination with $10-15 \%$ cold work area reduction after the co-extrusion.

The final cable has a small keystoning that compensates the trapezoidal deformation acquired during the coil winding.

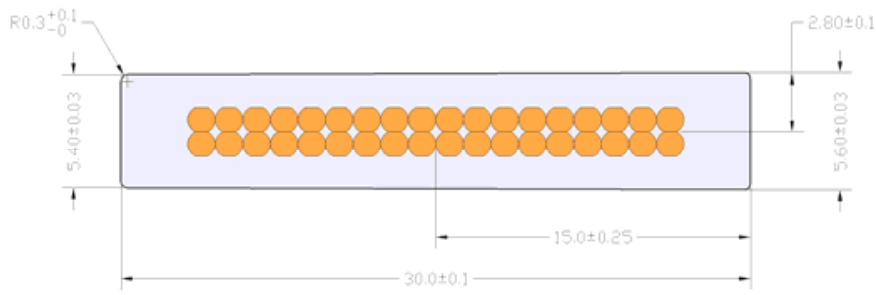

Fig. 1. Cable cross-section with dimensions in $[\mathrm{mm}]$ at $4.2 \mathrm{~K}$.

TABLE I

CABLE REQUIREMENTS AT 4.2K

\begin{tabular}{lcc}
\hline \hline \multicolumn{1}{c}{ Parameter } & Unit & Value \\
\hline Strand diameter & & $1.300 \pm 0.005$ \\
Number of strands & $\mathrm{mm}$ & 36 \\
Cable width & $\mathrm{mm}$ & $30.0 \pm 0.1$ \\
Cable minor edge thickness & $\mathrm{mm}$ & $5.60 \pm 0.03$ \\
Cable major edge thickness & $\mathrm{kA}$ & $>75.5$ \\
Cable critical current at 5T, 4.2K & $\mathrm{kA}$ & $>10.0$ \\
Cable critical current at 5.6T, 6.5K & $\mu \mathrm{m}$ & $<40$ \\
NbTi filament diameter & - & $>100$ \\
RRR of Cu matrix & - & $>800$ \\
RRR of Al stabilizer & - & $0.95 \pm 0.05$ \\
Strand Cu/non-Cu ratio & $\mathrm{MPa}$ & $>80$ \\
0.2\% yield strength of Al stabilizer & $\mathrm{MPa}$ & $>50$ \\
Shear strength of Al-Cu bond & & \\
\hline
\end{tabular}

\section{B. Insulation}

The PS employs a composite cable insulation made of polyamide and pre-preg glass tapes. This type of insulation, 
originally developed for the TOPAZ detector solenoid at KEK, was also used in the ATLAS Central Solenoid [10]. The cable is insulated with two layers of composite tape consisting of $25 \mu \mathrm{m}$ of a semi-dry epoxy on one side of $25 \mu \mathrm{m}$ Kapton tape and $75 \mu \mathrm{m}$ of pre-preg E-glass on the other side.

All gaps between turns and layers are to be filled with epoxy resin during vacuum impregnation. Since the composite cable insulation is impermeable for epoxy, sheets of dry Eglass are introduced between the coil layers and between coil and support structure to form paths for epoxy penetration.

The ground insulation between the cables and the support structure with the total thickness of $2 \mathrm{~mm}$ consists of dry Eglass and 2x25 $\mu \mathrm{m}$ layers of Kapton. That extra thickness of E-glass between the coil and support structure allows for machining of the outer coil surface after the impregnation for precise fitting the surfaces of the support shells and the coils.

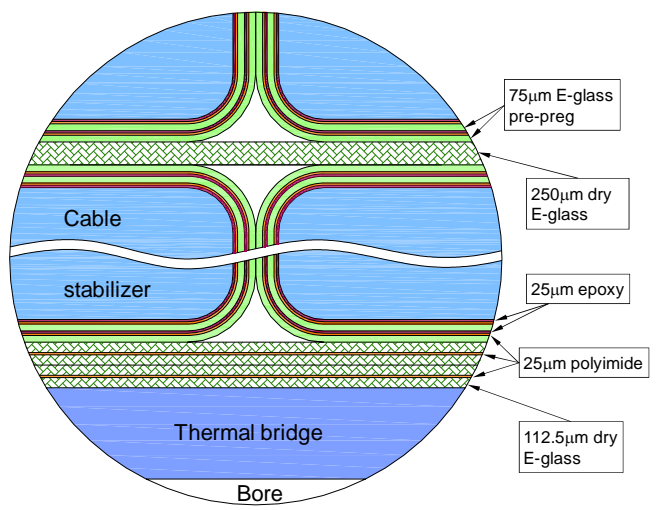

Fig. 2. Cable and interlayer insulation scheme.

\section{ELECTROMAGNETIC ANALYSIS}

\section{A. Magnetic design}

According to the requirements document [11], the PS magnet should have field gradient along the axis with the peak axial field of $4.6 \mathrm{~T}$ and the field at the interface with the Transport Solenoid (TS) [12] of 2.5-2.7 T. In order to fulfill this requirement, the magnet consists of three sections with 3 , 2 and 2 layers of the cable described in Fig. 1 and Table I, wound in the hard way around the aperture.

All coils are powered in series with $9.2 \mathrm{kA}$ current from the main power supply that corresponds to $4.6 \mathrm{~T}$ peak axial field. In addition to that, there is a trim power supply rated at $\pm 1 \mathrm{kA}$ connected across the 3-layer and middle 2-layer coils that allows changing the peak axial field by $\pm 0.5 \mathrm{~T}$ (and consequently the axial field gradient) with a minimal effect on the field at the TS interface that must remain the same irrespectively of the peak field. Although not imposed by the physics requirements, this additional field correction would help to continue running the experiment in case of unexpected degradation of the magnet performance.

Fig. 3 shows the FEM magnet model with the flux density diagram, and the flux density along the magnet axis. Note that the pictures also show the straight section of the TS, included in the model for the field-matching purposes, and the Heat and Radiation Shield (HRS) [13] made from C63200 bronze with the nominal relative magnetic permeability of 1.04 and resistivity of $2.46 \times 10^{-7} \Omega \mathrm{m}$. That specific material has been selected after industry studies and a cost optimization as a compromise between large resistivity, low magnetic permeability and forge-ability in sufficiently large pieces.

In order to guarantee meeting the peak field requirement of 4.6 $\mathrm{T}$, the magnet is designed to operate with a maximum $5.0 \mathrm{~T}$ on the axis while meeting all other requirements specified in [11]. The magnet cross-section and the field distribution are shown in Fig. 3.

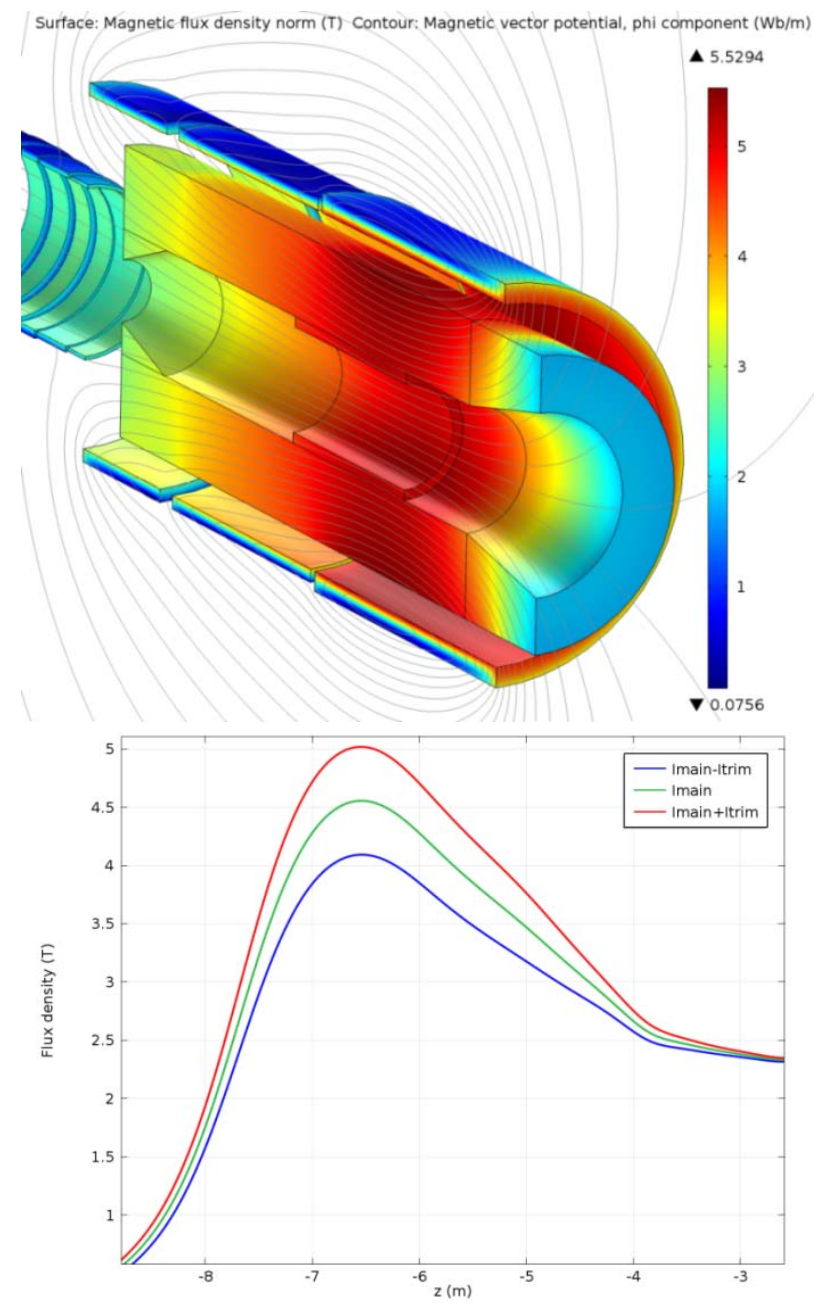

Fig. 3. Magnet cross-section with the flux density diagram (top) and the flux density along the magnet axis (bottom).

\section{B. Parameters}

The magnet is designed to operate at $64 \%$ of the Short Sample Limit (SSL) along the load line at $5.0 \mathrm{~T}$ axial field and 4.6 $\mathrm{K}$ as shown in Table II. At higher temperatures, the SSL shifts closer to the operating point and passes it at the temperature of $6.5 \mathrm{~K}$ - that is the current-sharing temperature at the operating current corresponding to $5.0 \mathrm{~T}$ axial field. In order to maintain the temperature margin requirement of $1.5 \mathrm{~K}$ at that field, the coil temperature shall not exceed $5.0 \mathrm{~K}$. In such case, the magnet operates at $68.9 \%$ with respect to the SSL at that temperature. Reducing the axial field to the nominal value of $4.6 \mathrm{~T}$, gains an additional $0.27 \mathrm{~K}$ margin. 
TABLE II

MAGNET PARAMETERS. THE DATA RANGES CORRESPOND TO VARIATION OF THE TRIM CURRENT.

\begin{tabular}{lcc}
\hline \multicolumn{1}{c}{ Parameter } & Unit & Value \\
\hline Liquid helium temperature $\left(\mathrm{T}_{\mathrm{LHe}}\right)$ & $\mathrm{K}$ & 4.6 \\
Operating current $\left(\mathrm{I}_{\mathrm{op}}\right)$ & $\mathrm{kA}$ & $9.20 \pm 0.95$ \\
Peak axial field at $\mathrm{I}_{\mathrm{op}}$ & $\mathrm{T}$ & $4.56 \pm 0.46$ \\
Peak coil field at $\mathrm{I}_{\mathrm{op}}$ & $\mathrm{T}$ & $4.97 \pm 0.51$ \\
Quench current at $\mathrm{T}_{\mathrm{LHe}}$ & $\mathrm{kA}$ & 15.81 \\
Current sharing temperature at $\mathrm{I}_{\mathrm{op}}$ & $\mathrm{K}$ & $7.04-6.50$ \\
Minumum temperature margin & $\mathrm{K}$ & 1.50 \\
Maximum allowable temperature $\left(\mathrm{T}_{\text {maxall }}\right)$ & $\mathrm{K}$ & $5.54-5.00$ \\
Fraction of SSL at $\mathrm{T}_{\mathrm{LHe}}$ & & $0.523-0.642$ \\
Fraction of SSL at $\mathrm{T}_{\text {maxall }}$ & & $0.628-0.689$ \\
Stored energy & $\mathrm{MJ}$ & $55.15-79.74$ \\
Self-inductance & $\mathrm{H}$ & 1.58 \\
Peak coil voltage & $\mathrm{V}$ & 600 \\
MQE at the peak field location & $\mathrm{mJ}$ & \\
Fast dump resistance & $\mathrm{m} \Omega$ & 59.11 \\
Initial time constant of a fast discharge & $\mathrm{s}$ & 26.7 \\
Warm bore diameter & $\mathrm{m}$ & 1.50 \\
Cold mass inner diameter & $\mathrm{m}$ & 1.70 \\
Cold mass length & $\mathrm{m}$ & 4.02 \\
Cold-mass weight & tonnes & 11.0 \\
\hline \hline
\end{tabular}

\section{Forces}

During the normal operation of the experiment, the axial force on the PS coil acts towards the TS. The axial and transverse force sensitivity due to possible coil misalignments was studied using the OPERA 3D model that also included the complete TS and DS coils. The forces summarized in Table III were calculated at the nominal coil position, as well as during the coil displacement in each of the three directions.

In case of the PS coil displacement in the transverse direction vs. the TS, the direction of the additional force is opposite to the direction of the displacement (i.e. the PS and TS coils tend to self-align). In case of the PS coil displacement in the axial direction vs. the TS, the extra force is in the same direction as the force at the nominal position.

The interaction of the magnetic field with the HRS magnetic permeability exerts an additional force on the coil during the normal operation. The direction of that force is opposite to the direction of the main force listed in Table III, and the magnitude is $\sim 12 \%$ of that. However, during the commissioning of the magnet system without the HRS installed, the cold mass supports will see the full PS-TS force.

During the standalone PS operation with the HRS installed, necessary for the correct field mapping, the axial force on the PS coil will be away from the TS. If a fast magnet discharge is initiated (due to quench or power failure), the eddy currents generated in the HRS will interact with the decaying magnetic field and exert an additional force on the cold mass of the same direction as the static force due to the HRS permeability.

The additional forces on the cold mass due to interaction with the HRS are summarized in Table IV at the nominal values of magnetic permeability and electrical resistivity. Since the C63200 bronze is not an electro-technical material, there is a relatively large variation of the chemical composition that may affect these quantities. A worst-case estimate of variation is included in Table IV as well.
TABLE III

STATIC LORENTZ FORCES ON THE PS COIL DUE TO INTERACTION WITH THE TS AT THE MAXIMUM DESIGN FIELD

\begin{tabular}{ccc}
\hline \hline $\begin{array}{c}\text { Force } \\
\text { component }\end{array}$ & $\begin{array}{c}\text { At the nominal } \\
\text { position, } \mathrm{kN}\end{array}$ & $\begin{array}{c}\text { Sensitivity to the coil } \\
\text { displacement, kN/cm }\end{array}$ \\
\hline $\mathrm{F}_{\mathrm{x}}$ & -15.7 & -9.0 \\
$\mathrm{~F}_{\mathrm{y}}$ & 0.0 & 18.0 \\
$\mathrm{~F}_{\mathrm{z}}$ & 1361.9 & 1.9 \\
\hline \hline
\end{tabular}

TABLE IV

PEAK DYNAMIC FORCE ON THE PS COLD MASS DUE TO INTERACTION WITH THE HRS DURING THE FAST DISCHARGE

\begin{tabular}{ccccc}
\hline \hline \multirow{2}{*}{$\begin{array}{c}\text { Peak field } \\
\text { on the axis, } \mathrm{T}\end{array}$} & \multicolumn{2}{c}{$\mathrm{F}_{\mathrm{z}}, \mathrm{kN}$ at the HRS resistivity of and permeability of } \\
\cline { 2 - 5 } & \multicolumn{2}{c}{$2.46 \times 10^{-7} \Omega \mathrm{m}$} & \multicolumn{2}{c}{$1.00 \times 10^{-7} \Omega \mathrm{m}$} \\
\hline & 1.04 & 1.10 & 1.04 & 1.10 \\
\hline 4.10 & -82 & -184 & -101 & -192 \\
4.55 & -76 & -167 & -91 & -173 \\
5.00 & -68 & -146 & -80 & -152 \\
\hline \hline
\end{tabular}

\section{STRUCTURAL ANALYSIS}

\section{A. Cold mass}

The coils are supported against Lorentz forces by the external shells made of Al 5083-O. Each of the three coils with its support shell forms a separate module. The three modules are bolted together through the end flanges that constitute the cold mass assembly.

The structural analysis was performed using the average coil properties defined by the FEM cable stack models [4]. It was assumed that all gaps between the coils and the support shell are filled with epoxy. Consequently, the magnet model was created with zero interference between coils and shells at the room temperature. The coil modules are to be assembled with layers of mica paper between the coils and the end flanges to allow sliding at these interfaces. The model included frictionless contact elements at these locations.

Fig. 4 shows the cold mass deformations after cooling down and energizing with the maximum current and the equivalent stress distribution. The peak equivalent stresses are $21 \mathrm{MPa}$ in the coil and $51 \mathrm{MPa}$ in the support structure after the cooldown. Energizing the coil with the maximum operating current brings the stresses to $73 \mathrm{MPa}$ and $96 \mathrm{MPa}$ for the coil and the support structure, respectively, which are within the maximum allowable values for all materials [4].

\section{B. Cold mass suspension system}

The PS cold mass suspension consists of axial and radial support systems for both the coil and thermal shield assemblies. The maximum axial force on the cold mass is 1.4 MN (Table III) towards the TS that occurs when both magnets are on. The axial force reverses the direction when the magnet is energized in a stand-alone mode and has a maximum of $-0.2 \mathrm{MN}$ (Table IV) when a fast discharge is initiated. The weight of the cold mass is estimated to be 11.0 tonnes (Table II) and that of the thermal shield is $\sim 1$ tonne. The cold mass suspension system is shown in Fig. 5.

In order to minimize the length of any penetration out the end of the vacuum vessel, the PS axial anchors made of Inconel 718 run the entire length of the cryostat. 


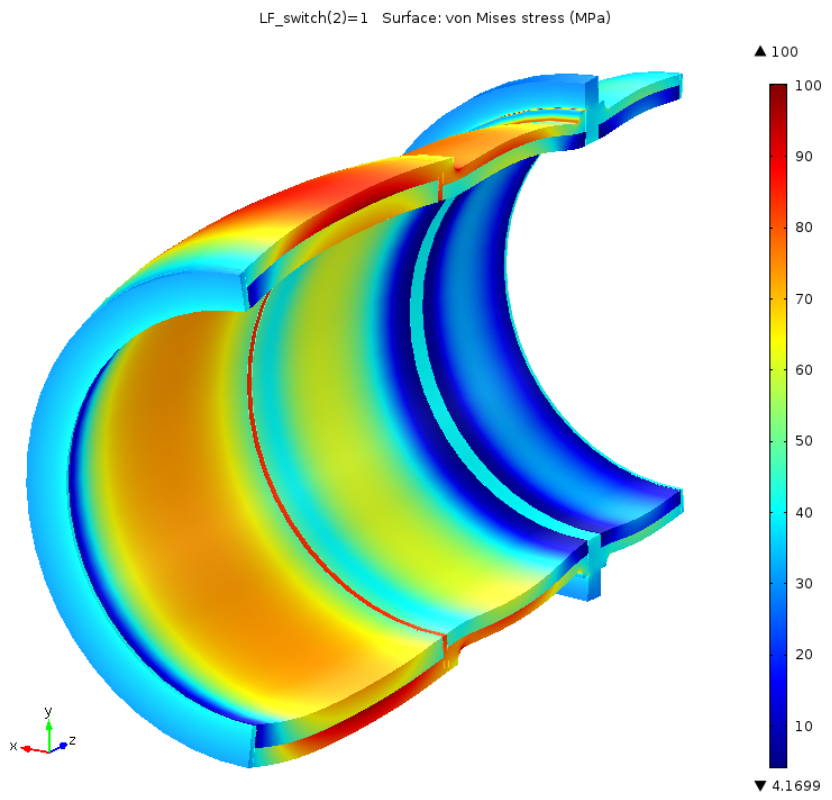

Fig. 4. Equivalent stress distribution in the cold mass after cooling down to 4.6 K and energizing to $5.0 \mathrm{~T}$ peak axial field. Note: deformations are multiplied by a factor of 100 .

Twelve axial rods, six running from each end of the vacuum vessel and attaching to the spacer ring between $2^{\text {nd }}$ and $3^{\text {rd }}$ coil modules constitute the axial anchor system. A series of Belleville springs at the vacuum vessel end of each rod accommodate approximately $3 \mathrm{~mm}$ of axial contraction of the rods themselves during the cooldown. Each set of six rods resists axial loads in one of the two directions. In both cases, the set not in use slides, unloading the springs, rather than allowing the rods to go into compression. Since the maximum load away from the TS is $\sim 14 \%$ of that towards the TS, the cross-section of the rod set at the TS end is proportionally smaller.

The radial supports resist the magnet weight and any transverse Lorentz forces. The assumed maximum gravity load is $2 \mathrm{~g}$ 's or 22 tonnes. As with the axial support, Inconel 718 was chosen for a series of 16 tension rods arranged in pairs at each end of the cold mass. The coil end is attached by a spherical clevis and a spherical washer pair is used at the vacuum vessel end. Since both ends of each rod are fixed, the main load is thermally induced during the cooldown. The thermal shield is supported by a similar set of radial rods. Table V lists the sizes and loads of the suspension rods.

\section{CONCLUSION}

The conceptual design of PS magnet has been reported. The magnet cold mass consists of three coil modules, bolted together and installed within a common cryostat. The necessary field profile is achieved by using different number of layers in the coil modules and a trim power supply.

The electromagnetic analysis performed with the actual properties of HRS shows that it has non-negligible effect on the forces acting on the cold mass. Depending on the operating regime, the axial force changes the direction that is reflected in the design of the cold mass supports.

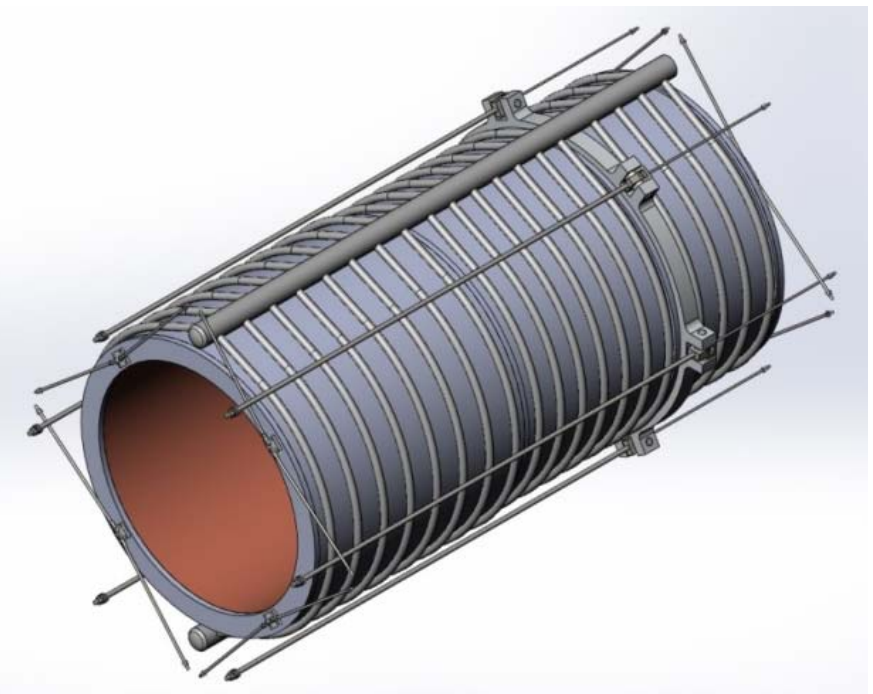

Fig. 5. Cold mass suspension system.

TABLE V

SUSPENSION COMPONENT SIZES AND LOADS

\begin{tabular}{lcccc}
\hline \multirow{2}{*}{ Parameter } & \multicolumn{2}{c}{ Axial } & \multicolumn{2}{c}{ Radial } \\
& non-TS end & TS end & Cold mass & Th. shield \\
\hline Rod diameter, mm & 28.6 & 12.7 & 15.9 & 15.9 \\
Peak stress, MPa & 360 & 255 & 270 & 245 \\
Total heat load, W & 1.31 & 0.86 & 4.48 & 11.84 \\
\hline \hline
\end{tabular}

\section{REFERENCES}

[1] M. J. Lamm, et al., "Solenoid Magnet System for the Fermilab Mu2e Experiment," IEEE Transactions on Applied. Superconductivity, Vol 22, No. 3, June 2012, pp.4100304.

[2] N. Mokhov, et al., "Beam-Induced Effects and Radiological Issues in High-Intensity High-Energy Fixed Target Experiments," presented at ICRS12, Progress in Nuclear Science and Tech., to be published.

[3] V. V. Kashikhin et al., "Conceptual Design of the Mu2e Production Solenoid Cold Mass," Advances in Cryogenic Engineering, AIP Conf. Proc., 1434, 893-900 (2012).

[4] V. V. Kashikhin, et al., "Design Studies of Mu2e Production Solenoid," Mu2e-doc-1110.

[5] A. Yamamoto, et al.,, "Design and Development of the ATLAS Central Solenoid Magnet," IEEE Transactions on Applied Superconductivity, Vol. 9, No. 2, June 1999, pp.852-855.

[6] G. Baccaglioni, et al., "Production and Qualification of $40 \mathrm{~km}$ of AlStabilized NbTi Cable for the ATLAS Experiment at CERN," IEEE Transactions on Applied Superconductivity, Vol. 12, No. 1, March 2002, pp.1215-1218.

[7] B. Blau, et al., "The CMS Conductor," IEEE Transactions on Applied Superconductivity, Vol. 12, No. 1, March 2002, pp.345-348.

[8] K. Wada, et al., "Development of High-Strength and High RRR Aluminum-Stabilized Superconductor for the ATLAS Thin Solenoid," IEEE Transactions on Applied Superconductivity, Vol. 10, No. 1, March 2000, pp.373-376.

[9] K. Wada, et al., "High-Strength and High-RRR Al-Ni Alloy for Aluminum-Stabilized Superconductor," IEEE Transactions on Applied Superconductivity, Vol. 10, No. 1, March 2000, pp.1012-1015.

[10] ATLAS Central Solenoid Technical Design Report, ATLAS Magnet Project TDR Vol. 4, April 1997.

[11] M. Lamm, "Mu2e Production Solenoid Requirements Document," Mu2e-doc-945.

[12] G. Ambrosio et al., "Design of the Transport Solenoid for the Mu2e experiment," IEEE Transactions on Applied Superconductivity, submitted for publication.

[13] R. Coleman, et al., "Requirements for the Mu2e Production Solenoid Heat and Radiation Shield,” Mu2e-doc-1092. 